\title{
SURFACE POTENTIAL INVESTIGATIONS OF INDIUM PHOSPHATE REAL SURFACES BY MEANS OF TRANSVERSE ACOUSTOELECTRIC METHOD
}

\author{
T. Pustelny \\ Institute of Physics, Silesian Technical University \\ Krzywoustego 2, 44-100 Gliwice, Poland
}

(Received January 3, 1995; revised version May 10, 1995;

in final form August 16, 1995)

A method of surface potential determination in semiconductors by means of the transverse acoustoelectric effect is described. The results of theoretical analysis of the transverse acoustoclectric voltage versus surface potential and different surface electrical parameters in indium phosphate single crystals are presented. The experimental results of the surface potential investigations have been obtained after various surface treatments in $\operatorname{In} P(110)$ and InP(100) crystals. A strong influence of the chemical and mechanical surface treatments upon the surface potential values has been observed from the measurements. The surface $\operatorname{In} P(110)$ was more sensitive to different surface treatments. The changes of the surface potential values were about two times greater for $\operatorname{In} P(110)$ than for $\operatorname{In} P(100)$ samples. The surface potentials after surface treatments obtained by the acoustics method were of the range $-0.08[\mathrm{~V}]$ to $-0.22[\mathrm{~V}]$.

PACS numbers: $73.20 .-r, 72.50 .+b, 43.35 .+d$

\section{Introduction}

The electrical and electronic properties of the real semiconductor surface may be determined by the surface electrical potential, the type of impurity atoms and molecules, their concentration and location in the energy gap in the target material, the surface mobility of carriers, the time of majority and minority carriers and the carrier trapping velocity by fast and slow electronic surface states [1]. The surface potential is an especially important parameter in the clectronic devices technology, which affects strongly their electrical properties [2].

Indium phosphate is one of the most popular material from the III-V semiconductors group. The semiconductors of this group are very important in the 
electronic devices technology. InP has a very interesting optical property. Its high carrier mobility gives the possibility to construct the electronic devices working in high frequency range, e.g. very high frequency amplifiers and generators. The InP(100) crystals are used as a target material for molecular epitaxy techniques. Indium phosphate is often used in different variety sensor applications [3].

The so-called real surface of semiconductor is a surface obtained after cutting, polishing, grinding and standard chemical etching of the crystal. Such surface appears at different steps of the semiconductor devices technology. Up to now, only a few works studied the real $\operatorname{InP}(110)$ and $\operatorname{InP}(100)$ surfaces [4-6].

Recently more and more interest of using the surface acoustic waves for semiconductor surface investigations has been observed. Surface acoustic methods are based on the interaction between the surface acoustic wave of the Rayleigh type and free carriers in the semiconductor in the layered structure of the piezoelectric wave-guide-semiconductor [7]. The methods use the propagation of the surface acoustic wave of the high frequency, thus it is possible to determine the dynamic values of investigated parameters of the studied semiconductor surface.

This paper presents the new and original experimental results of surface potential investigations in $\operatorname{In} P(110)$ and $\operatorname{InP}(100)$ crystals obtained by the surface acoustic wave method.

\section{Experimental}

If the surface acoustic wave (SAW) propagates in the piezoelectric-semiconductor structure (Fig. 1), then the electric field accompanying the wave penetrates a near-surface region of the semiconductor. The penetration depth of the electric field inside the semiconductor is of the order of the semiconductor extrinsing Debye length or the acoustic wavelength (whichever is shorter). The electric field changes the free carriers concentration in the near-surface region of semiconductor and causes the drift of these carriers [8]. In Ref. [9] there are described different aspects of the interactions between surface acoustic wave and electric carriers in the piezoelectric-semiconductor layered structure. The difference of electric poten-

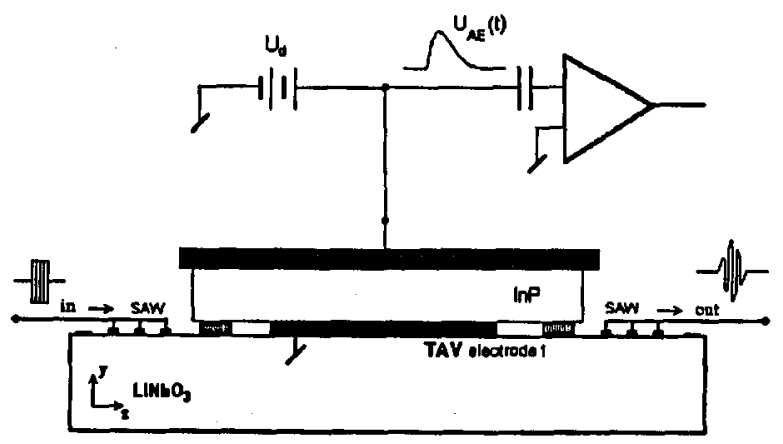

Fig. 1. The layered structure: piezoelectric wave guide and investigated semiconductor. 
tial between the semiconductor surface and its bulk (i.e. transverse acoustoelectric voltage, TAV) as a result of the electric carrier and the surface acoustic wave interaction may be observed [10-12]. The results of theoretical analysis of the transverse acoustoelectric effect in Refs. [11, 13] were presented. These results made a theoretical ground for a new acoustic method of the surface potential determination in semiconductors [14].

The important advantage of this method is that it does not require the ohmic contacts with the investigated semiconductor sample. The ohmic contacts production for the III-V semiconductors group is a difficult technological process, different for each type of semiconductor. After this process the surface changes the electrical and electronic parameters significantly.

The TAV method does not require ohmic contact and it can be used for investigations of the real properties of $\operatorname{In} P(110)$ and $\operatorname{In} P(100)$ surfaces submitted to different mechanical and chemical surface treatments. The TAV method was used to investigate the $\operatorname{InP}(110)$ and $\operatorname{In} P(100)$ single crystal samples with the following volumetric parameters:

$\operatorname{InP}(110)$ :

- $n$-type electrical conductivity

- carriers mobility: $\mu_{\mathrm{nb}}=0.420\left[\mathrm{~m}^{2} /(\mathrm{V} \mathrm{s})\right], \mu_{\mathrm{pb}}=0.016\left[\mathrm{~m}^{2} /(\mathrm{V} \mathrm{s})\right]$

- permittivity: $\varepsilon=10.1[-]$

- band gap: $E_{\mathrm{g}}=1.25[\mathrm{eV}]$

- electron concentration: $N_{\mathrm{d}}=3.1 \times 10^{20}\left[\mathrm{~m}^{-3}\right]$

- resistivity: $\rho=8.0 \times 10^{3}[\Omega \mathrm{m}]$ tively;

- effective mass of holes and electrons $m_{\mathrm{p}}^{*}=0.04$ and $m_{\mathrm{n}^{\prime}}^{*}=0.03$, respec$\operatorname{InP}(100)$ :

- $n$-type electrical conductivity

- carriers mobility: $\mu_{\mathrm{nb}}=0.400\left[\mathrm{~m}^{2} /(\mathrm{V} \mathrm{s})\right], \mu_{\mathrm{pb}}=0.017\left[\mathrm{~m}^{2} /(\mathrm{V} \mathrm{s})\right]$

- permittivity: $\varepsilon=9.7[-]$

- band gap: $E_{\mathrm{g}}=1.27[\mathrm{eV}]$

- electron concentration: $N_{\mathrm{d}}=1.1 \times 10^{20}\left[\mathrm{~m}^{-3}\right]$

- resistivity: $\rho=1.5 \times 10^{4}[\Omega \mathrm{m}]$ tively.

- effective mass of holes and electrons $m_{\mathrm{p}}^{*}=0.04$ and $m_{\mathbf{n}^{\prime}}^{*}=0.03$, respec-

The electrical properties of the semiconductor surface and the depth of the interaction between the electric field from SAW and electrical carriers in the near-surface region can be changed during the experimental investigations by e.g. applying the external perpendicular electrical voltage $U_{\mathrm{d}}$ across the semiconductor sample.

The experimental procedure of the layered structure, piezoelectric wave guide and investigated semiconductor using the transverse acoustoelectric effect was presented in Ref. [13]. Rf pulse of $194 \mathrm{MHz}$ and about $2 \mu$ s duration was applied to the input transducer to generate the surface acoustic wave on $Y$-cut, $Z$-propagating in $\mathrm{LiNbO}_{3}$ delay line. The experimental set-up was described in Ref. [15]. 


\section{Results and discussion}

The main purpose of the investigations was exploration of the influence of the surface treatments for the surface electric potential. The InP samples were measured after some mechanical and chemical surface treatments, typical in the technology of electronic devices. The surface parameters were tested after mechanical grinding by alumina powders with various granulation of grains and after polishing with diamond paste. The surface parameters was also tested after cleaning the samples with acetone, methanol, benzene and after chemical etching HF or in $3 \mathrm{IINO}_{3}+10 \mathrm{H}_{2} \mathrm{O}_{2}$ acids. The samples were rinsed in methanol and deionized water after the treatments. Various combinations of simultaneously mechanical and chemical treatments were applied.

The amplitude $U_{\mathrm{AE}}$ of transverse acoustoclectric voltage changes after the surface treatments. It results mainly due to the change of the density of electronic surface states. The time shape of the TAV signal changes, too. The changing of the time shape of acoustoelectric voltage is a result of the alterations of the minority carriers life time in the near-surface region $[16,17]$. Using the TAV effect, the method of the determination of the minority carriers life time was proposed. The results of the life time investigations in the III-V semiconductors group were presented in Refs. [8, 17]. Different intensity of the changes in $U_{\mathrm{AE}}$ amplitudes in InP samples were observed very clearly after various surface treatments. The amplitudes $U_{\mathrm{AE}}$ changed themselves for some hours until they reached a steady state. The time settled depends on the type of surface treatment as well as on the means of washing and cleaning the semiconductor samples.

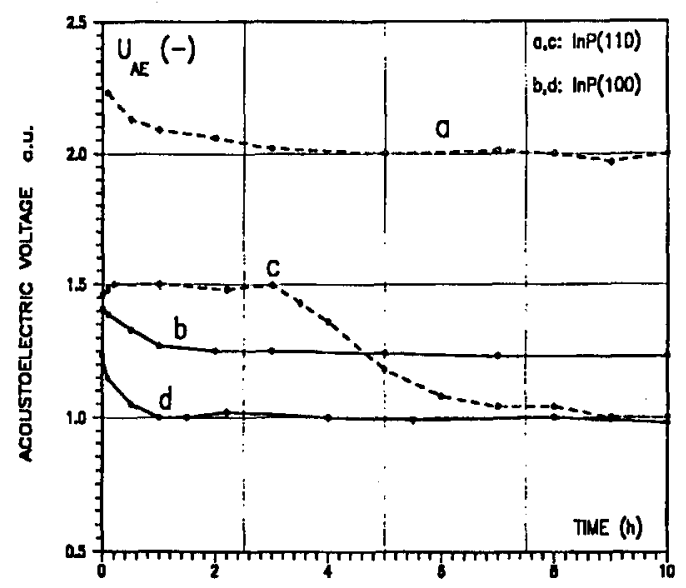

Fig. 2. The amplitude $U_{\mathrm{AE}}$ after different surface treatments: diamond paste polishing in: (a) InP(110), (b) $\operatorname{In} \mathrm{P}(100)$; etching in $\mathrm{INO}_{3}$ acid in: (c) $\operatorname{In} \mathrm{P}(110)$, (d) $\operatorname{InP}(100)$.

Figure 2 presents TAV amplitudes $U_{\mathrm{AE}}$ versus time after surface treatments in $\operatorname{InP}(110)$ and in $\operatorname{InP}(100)$ for two cases: for the diamond paste polishing and for the etching in $\mathrm{HNO}_{3}$. The amplitudes $U_{\mathrm{AE}}$ are presented in arbitrary units (a.u.), 
they are related to the value $U_{\mathrm{AE}}$ obtained after $\mathrm{IINO}_{3}$ acid etching, after about 10 hours in air atmosphere and for external voltage $U_{\mathrm{d}}=0$ [V].

The steady states of the $U_{\mathrm{AE}}$ amplitudes were reached after about 6-7 hours for $\mathrm{HNO}_{3}$ acids etching. A similar situation was observed in the case of IIF acid etching, too. In InP after diamond paste polishing these periods of time were from 0.5 to 3 hours. For InP surfaces grinded by alumina powder, a great intensity of changes and a long time of $U_{\mathrm{AE}}$ alterations were observed. However, these effects were smaller than after $\mathrm{INO}_{3}$ acid etching. In InP(100) samples the changes were essentially smaller, about some tens per cent. Also the settled times of the surface potential were shorter in $\operatorname{InP}(100)$ than in $\operatorname{InP}(110)$ samples, for all cases.

The smallest TAV amplitudes were observed after alumina powder grinding. They were about four times smaller than after diamond paste polishing and about one and half times smaller than after IIF and $\mathrm{INNO}_{3}$ acids etching. It follows mainly from the fact that after alumina grinding the investigated surfaces were mechanically more defected. The density of surface states is then the highest. The cleaning and rinsing InP samples in benzene or acetone changed the $U_{\mathrm{AE}}$ amplitude a little, about some per cent.

In Fig. 3. the experimental dependences of the $U_{\mathrm{AE}}$ amplitudes versus the external voltage $U_{\mathrm{d}}$ for $\operatorname{In} \mathrm{P}(110)$ are shown. The characteristic (a) was obtained for the sample polished by diamond paste. The characteristics (b) after about 1 minute HF etching and (c) after grinding by alumina powder No. 100 were determined. In all cases, after surface treatments the samples were washed in methanol and rinsed in deionized water. The characteristics in Fig. 3. were obtained about 20 hours after the surface treatments. The $U_{\mathrm{AE}}$ amplitudes are in arbitrary units. The value $U_{\mathrm{AE}}=1[-]$ relates to $U_{\mathrm{d}}=0[\mathrm{~V}]$ for each case. Clearly, the amplitudes $U_{\mathrm{AE}}$ have different values in absolute units. The steady state amplitude $U_{\mathrm{AE}}$ for the case of

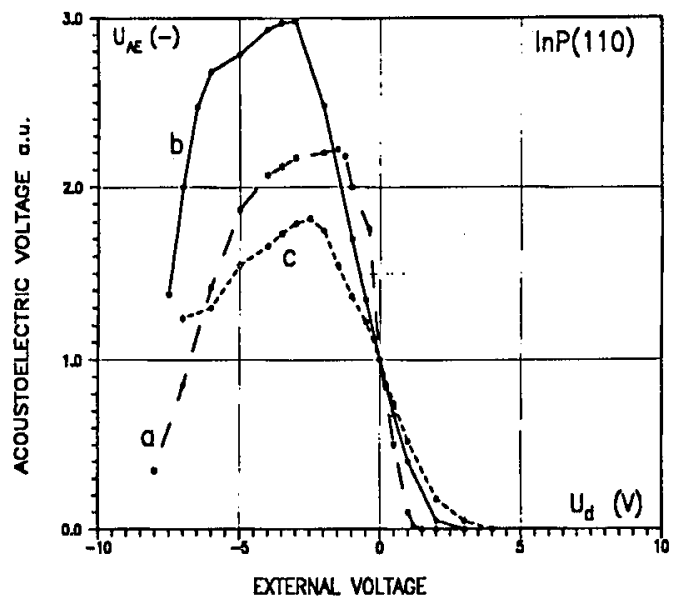

Fig. 3. The experimental dependence of $U_{\mathrm{AE}}$ external voltage $U_{\mathrm{d}}$ after (a) diamond paste polishing, (b) etching in $\mathrm{HF}$ acid, (c) alumina powder grinding. 
diamond paste polishing obtained after a few hours was about twice as great as $U_{\mathrm{AE}}$ after $\mathrm{IINO}_{3}$ etching.

It is shown in Ref. [18] that in the case of good polishing of semiconductor surface, the coefficient of the carriers diffusive dispersion $k_{\mathrm{d}}$ which characterises the charge carriers reflection from the semiconductor surface is approximately equal to zero because the reflection is of the mirror image type. For a strongly mechanically defected surface the carriers reflections have a diffusive character, so $k_{\mathrm{d}}$ is approximately equal to one, $k_{\mathrm{d}} \approx 1$.

For the theoretical analyses the following values of $k_{\mathrm{d}}$ were taken:

(i) $k_{\mathrm{d}}=0.0$ for splitting surface,

(ii) $k_{\mathrm{d}}=0.1$ for polishing surface with diamond paste,

(iii) $k_{d}=0.8$ for $\mathrm{HNO}_{3}$ acid etching,

(iiii) $k_{\mathrm{d}}=0.9$ for HF acid etching and

(iiiii) $k_{\mathrm{d}}=1.0$ for alumina powder No. 100 grinding.

In Fig. 4 the theoretical dependences $U_{\mathrm{AE}}=f\left(U_{\mathrm{S}}\right)$ for $\operatorname{InP}(110)$ and $\operatorname{InP}(100)$ samples are presented. These theoretical dependences were obtained numerically using theoretical equations given in Ref. [16]. In the calculations the $\operatorname{InP}(100)$ and $\operatorname{InP}(110)$ volumetric values of carriers mobility, permittivity and conductivity were taken as presented above and the coefficient $k_{\mathrm{d}}=0.1$. The potential $U_{\mathrm{S}}$ is the arbitrary value of the surface potential, $U_{\mathrm{S}}=e \Phi_{\mathrm{S}} / k T$ and $\Phi_{\mathrm{S}}[\mathrm{V}]$ is the surface potential in semiconductors.

Using the experimental relation $U_{\mathrm{AE}}=f\left(U_{\mathrm{d}}\right)$ and the theoretical relation $U_{\mathrm{AE}}=f\left(U_{\mathrm{S}}\right)$ for the analysed sample (Figs. 3 and 4) one may propose the method for the determination of the surface potential value in semiconductors assuming for zero external bias voltage. This metlod was described in Ref. [19].

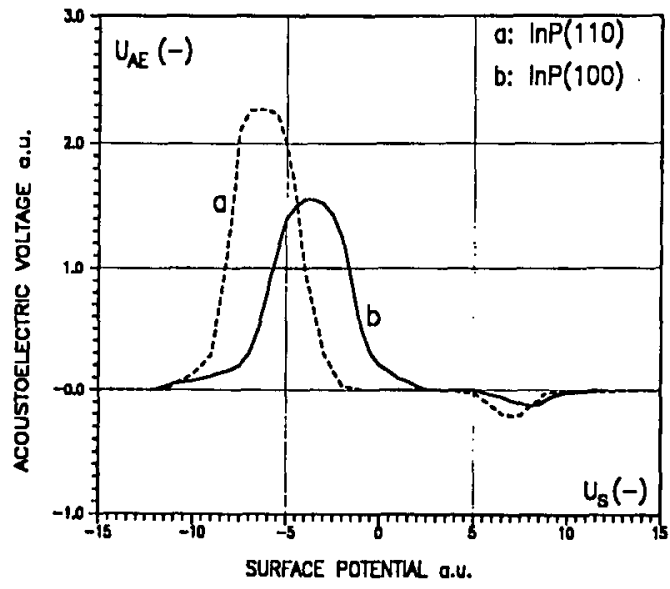

Fig. 4. The theoretical dependence of $U_{\mathrm{AE}}$ on surface potential $U_{\mathrm{S}}(\mathrm{a}) \operatorname{In} \mathrm{P}(110)$, (b) $\operatorname{InP}(100)$. 
For the investigated samples, the values of the surface potential $\Phi_{\mathrm{S}}$ obtained by this method were equal to for $\operatorname{InP}(110)$ :

(a) $\Phi_{\mathrm{S}}=-0.11 \pm 0.01[\mathrm{~V}]$ (after diamond paste polishing)

(b) $\Phi_{\mathrm{S}}=-0.22 \pm 0.01[\mathrm{~V}]$ (after IIF acid etching)

(c) $\Phi_{\mathrm{S}}=-0.18 \pm 0.01[\mathrm{~V}]$ (after alumina powder grinding) for $\operatorname{InP}(100)$ :

(d) $\Phi_{\mathrm{S}}=-0.08 \pm 0.01[\mathrm{~V}]$ (after diamond paste polishing)

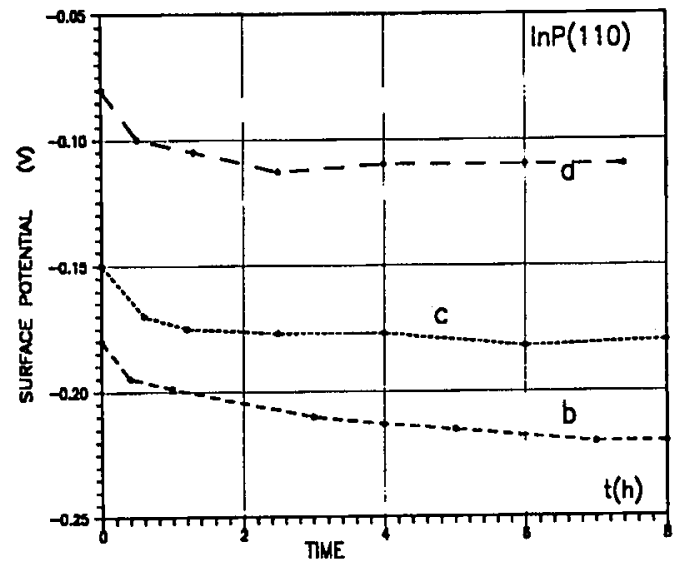

Fig. 5. Surface potential alterations after surface treatments in $\operatorname{In} P(110)$ : (a) diamond paste polishing, (b) HF acid etching, (c) alumina powder grinding.

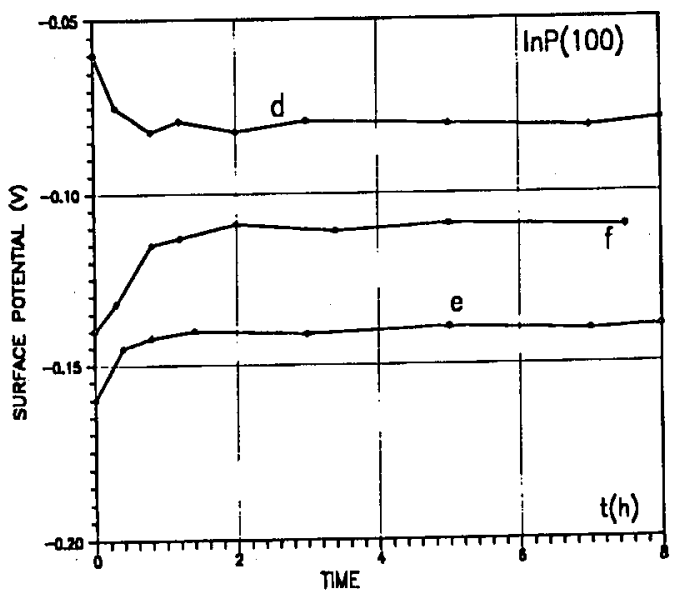

Fig. 6. Surface potential alterations after surface treatments in nip(100): (d) diamond paste polishing, (e) HF acid etching, (f) alumina powder grinding. 
(e) $\Phi_{\mathrm{S}}=-0.14 \pm 0.01[\mathrm{~V}]$ (after HF acid etching)

(f) $\Phi_{\mathrm{S}}=-0.11 \pm 0.01[\mathrm{~V}]$ (after alumina powder grinding).

These surface potential values were determined about ten hours after their surface treatments. The time alterations of surface potential after different surface treatments for $\operatorname{In} \mathrm{P}(110)$ and for $\operatorname{In} \mathrm{P}(100)$ are presented in Figs. 5 and 6 . The obtained results have shown that after various surface treatments the differences between the surface potential values may be as great as some tens per cent or even more.

\section{Conclusion}

All samples investigated by the TAV method were prepared from the same $\operatorname{In} \mathrm{P}(110)$ or $\operatorname{In} \mathrm{P}(100)$ crystals. They had high resistivity and the $n$-type electrical conductivity. After surface treatments the type of surface electrical conductivity did not change in any of the investigated samples. The surface conductivity of InP samples was in depletion states, but the $n$-type of conductivity was kept. It follows from the fact that in InP crystals the electron mobility is much higher than the hole mobility. For this reason it is difficult to change the surface electrical conductivity after surface treatments. However, the changes of the type of surface electrical conductivity were observed in high resistivity GaP crystals [6].

After alumina grinding and after $\mathrm{HF}$ or $\mathrm{HNO}_{3}$ acids etching the InP surfaces were strongly mechanically defected. The density of surface states was great and the interaction between them and atmosphere molecules (mainly oxygen molecules) had the long time character. It seems that washing with methanol and rinsing with deionized water after $\mathrm{HF}$ or $\mathrm{HNO}_{3}$ acids etching is not enough sufficient and effective. It is suspected that not exact washing and rinsing cause that InP has been etched by rest acids molecules for some hours after its surface treatment. To verify this suspicion, the InP sample was washed in methanol at about $50^{\circ} \mathrm{C}$ temperature after etching in $\mathrm{HF}$ acid. The $U_{\mathrm{AE}}$ amplitude stopped to change after a shorter time, about half an hour. It is expected to be much better to wash the InP sample in boiled benzene for about twenty minutes. The high temperature washing methods are used in silicon technology [20].

It is shown in Ref. [21] that the transverse acoustoelectric voltage method gives the possibility of high resistivity semiconductors investigations. For low resistivity and for very high resistivity semiconductor samples, the acoustoelectric signals are too small for the surface parameters determination. In such cases the surface parameters are determined with a very low accuracy. The similar situations are observed for all electrical semiconductor investigation methods $[22,23]$.

In the group of semiconductors surface methods, the transverse acoustoelectric voltage method seems to be a new and attractive way of the surface potential investigation. This method is not destructive. Moreover, it does not require the ohmic contacts to tested semiconductor samples. The TAV method gives the possibility to determine the values of the parameters in high and very high frequency ranges in InP crystals.

All presented results were obtained for $194 \mathrm{MHz}$ surface acoustic wave frequency. There is a possibility to investigate the surface semiconductor up to some $\mathrm{GHz}$ by using of the SAW techniques [16]. The surface potential values were deter- 
mined with an accuracy of some per cent. After surface treatments the electrical parameters have been changed for $\operatorname{InP}(110)$ surface less than for $\operatorname{InP}(110)$. It is difficult to obtain $\operatorname{InP}(100)$ surface because this surface is not cleaved in contrary to $\operatorname{InP}(110)$ surface. Despite this difficulty, InP(100) crystals are more and more often used as the substrates for the growth of epitaxial thin layers in semiconductor device technology [18].

The acoustic method presented above may be very useful, complementary to electrical, photo spectroscopy and photo emission methods [23] of high resistivity semiconductor surface investigations. In Refs. [12] and [15] the common experiments concerning the surface potential in GaP samples are presented by means of this acoustoelectric method and the surface photo voltage spectroscopy method. The results obtained from this method were the same with some per cent accuracy.

The additional motivation for InP investigations was the explanation of the possibility of using this material for luminescence temperature fiber optic sensor construction [24].

This work was sponsored by the State Committee for Scientific Research (Republic of Poland) within the grant 8 T11B 06009.

\section{Acknowledgment}

Author would like to thank prof. A. Opilski from Institute of Physics Silesian Technical University in Gliwice for helpful discussions and L. Węgierska for help in experimental measurements. Author would like to thank Prof. J. Bobitskij from the University of Technology in Lviv (Republic of Ukraine) for preparing the semiconductor materials for investigations.

\section{References}

[1] C.E. Reed, C. Scoh, Surface Physics of Phosphors and Semiconductors, Academic Press, London 1987.

[2] S.M. Sze, Physics of Semiconductor Devices, Wiley Press, New York 1989.

[3] T. Pustelny, Fiber Optic Temperature Sensors, EUROPTRODE II, Firenze (1994), p. 126.

[4] J.M. Moison, M. Bensoussan, Surf. Sci. 168, 68-73 (1986).

[5] J.M. Moison, Van Rampay, Appl. Phys. Lett. 48, 1362-1364 (1987).

[6] T. Pustelny, B. Adamowicz, J. Tech. Phys. 35, 201-208 (1994).

[7] M. Tabib-Azar, Solid-State Electronics 31, 1197-1204 (1988).

[8] B. Davari, J. Appl. Phys. 1, 54-56 (1983).

[9] I. Yakovkin, V. Vyun, in: Proc. Intern. Symp. "Surface Waves in Solid and Layered Structures", Vol. 1, Izd. Nauka, Novosibirsk, 1986, p. 183.

[10] Z. Kubik, Acoustic Letters 8, 20-24 (1984).

[11] J.H. Gilboa, M. Motamedi, P. Das, in: Proc. Ultrasonic Symp., IEEE, New York 1986, p. 663.

[12] Pustelny T., Adamowicz B., J. Tech. Physics 34, 299-309 (1993).

[13] T. Pustelny, Z. Kubik, Archives Acoustics, 19, 271-280 (1994). 
[14] T. Pustelny, in: Proc. 11th Intern. FASE Symp., FASE, Valencia 1994, p. 113.

[15] T. Pustelny, J. Tech. Physics 36, 17-28 (1995).

[16] T. Pustelny, Ultrasonic Measurement Technics in Science \& Practice 2, 54-63 (1994).

[17] T. Pustelny, Acustica, (1994), (in press).

[18] S. Peka, Semiconductor Surface Physics, Nauka, Kijev, 1991 (in Russian).

[19] Pustelny, Molecular and Quantum Acoustic. 14, 112-120 (1993).

[20] A. Rzanov, Ellektronnyje Processy na Powierchnosti Poluprovodnikov, Nauka, Moscow 1986 (in Russian).

[21] P. Das, M. Tabib-Azir, Appl. Phys. Lett. 51, 436-438 (1987).

[22] M.S. El-Dessouki, V. Attia, M.M. El-Deen.Saad, F. Gobrial, Phys. Stalus Solidi A 99, 99 (1987).

[23] J. Szuber, Appl. Surf. Science. 55, 143-147 (1992).

[24] T. Pustelny, Opto-Eleclranics Review, 4, 117-121 (1993). 